\title{
Fetal growth and gestational factors as predictors of schizophrenia in 22q11.2 deletion syndrome
}

\author{
Lily Van, BHSc ${ }^{1,2}$, Nancy J. Butcher, MSc ${ }^{1,3}$, Gregory Costain, MD, PhD ${ }^{1,2}$, \\ Lucas Ogura, BSc', Eva W.C. Chow, MD, FRCPC ${ }^{1,4}$ and Anne S. Bassett, MD, FRCPC $1,3-8$
}

\begin{abstract}
Purpose: Schizophrenia occurs in $20-25 \%$ of adults with 22 q11.2 deletion syndrome (22q11.2DS). General population studies of schizophrenia report associations with perinatal complications, although effect sizes are generally low. We aimed to determine whether such factors are associated with expression of schizophrenia in individuals with 22q11.2DS.
\end{abstract}

Methods: We investigated the relationship of small for gestational age (SGA) birth weight ( $<3$ rd percentile for sex and gestational age) and prematurity ( $<37$ weeks gestation) to expression of schizophrenia in a well-characterized cohort of 123 adults with 22q11.2DS. Outcome measures included adjusted odds ratios and positive and negative predictive values (PPV and NPV) for schizophrenia.

Results: SGA birth weight $(\mathrm{OR}=3.52,95 \% \mathrm{CI}=1.34-9.22)$ and prematurity $(\mathrm{OR}=5.38,95 \% \mathrm{CI}=1.63-17.75)$, but not maternal factors, were significant risk factors for schizophrenia in 22q11.2DS. Being born SGA or premature resulted in a PPV of $46 \%$ for schizophrenia; NPV in the absence of both features was $83 \%$. Post hoc analyses suggested these perinatal complications were also associated with factors indicative of increased severity of schizophrenia.

Conclusion: In 22q11.2DS, fetal growth and gestation may have a clinically significant impact on future risk for schizophrenia. These data advance our understanding of determinants of disease-specific expression in 22q11.2DS, with implications for other genomic disorders.

Genet Med advance online publication 18 June 2015

Key Words: copy-number variation; 22q11 deletion; genetic counseling; intrauterine growth restriction; prematurity

\section{INTRODUCTION}

22q11.2 deletion syndrome (22q11.2DS; OMIM 188400/192430) is a common but under-recognized microdeletion syndrome, and an established genetic subtype of schizophrenia. ${ }^{1-3}$ The associated 22q11.2 deletion occurs most commonly as a de novo mutation; inherited deletions are found in up to $10 \%$ of newly diagnosed cases. ${ }^{1,2}$ Penetrance is high for any observable phenotype, but expression is variable and unpredictable. ${ }^{1,2}$ About one in four individuals with 22q11.2DS develops schizophrenia in late adolescence or early adulthood. ${ }^{2,4,5}$ Identifying factors that may influence the 20- to 25-fold elevated risk for schizophrenia in 22q11.2DS is a matter of intense interest to patients, families, clinicians, and researchers. ${ }^{6}$

Multiple general population-based studies of schizophrenia have reported associations with adverse perinatal outcomes, especially small for gestational age (SGA) birth weight and late prematurity, ${ }^{7-10}$ although effect sizes are generally low. Similarly, cohort studies of infants with these perinatal features show elevated risk for neurodevelopmental sequelae in children. ${ }^{11,12}$ The mechanisms underlying these established associations are not well understood. Subtle brain injury and/or disruption of normal brain maturation, mediated by hypoxia, are often cited, and placental insufficiency may play a role. Fetal genotype and its likely interaction with antepartum factors however are rarely studied. ${ }^{13,14}$

Previous studies have found that 22q11.2 deletions may be associated with aberrant fetal growth and gestation. ${ }^{13,15-18}$ The degree to which these features influence expression of schizophrenia within 22q11.2DS is unknown. Using a retrospective nested case-control design in a well-characterized adult cohort with 22q11.2DS, we tested whether prematurity and a level of SGA birth weight most likely to represent the pathogenic process of intrauterine growth restriction (IUGR) were associated with expression of schizophrenia. We anticipated that the relative genetic homogeneity of this sample would increase effect sizes to the point of being clinically informative.

\section{MATERIALS AND METHODS}

\section{Subjects}

The subjects for this study were from an adult (aged $\geq 18$ years) Canadian cohort with 22q11.2DS.,15,19-24 All 22q11.2 deletions were confirmed with fluorescence in situ hybridization

\footnotetext{
${ }^{1}$ Clinical Genetics Research Program, Centre for Addiction and Mental Health, Toronto, Ontario, Canada; ${ }^{2}$ Undergraduate Medical Education, Faculty of Medicine, University of Toronto, Toronto, Ontario, Canada; ${ }^{3}$ Institute of Medical Science, University of Toronto, Toronto, Ontario, Canada; ${ }^{4}$ Department of Psychiatry, University of Toronto, Toronto, Ontario, Canada; ${ }^{5}$ Campbell Family Mental Health Research Institute, Centre for Addiction and Mental Health, Toronto, Ontario, Canada; ${ }^{6}$ Department of Psychiatry, Toronto General Research Institute, University Health Network, Toronto, Ontario, Canada; ${ }^{7}$ Division of Cardiology, Department of Medicine, University Health Network, Toronto, Ontario, Canada; ${ }^{8}$ The Dalglish Family Hearts and Minds Clinic for Adults with 22q11.2 Deletion Syndrome, University Health Network, Toronto, Ontario, Canada. Correspondence: Anne S. Bassett (anne.bassett@utoronto.ca)
} 
and/or chromosomal microarray analysis using standard probes and methods. ${ }^{19,22}$ The majority of subjects were ascertained through adult congenital cardiac, psychiatric, or genetics services as described previously. ${ }^{4,19}$ Written informed consent was obtained for all subjects, and the study was approved by local research ethics boards.

All subjects born in singleton pregnancies and with data available on both psychotic disorder outcome, and birth weight and gestational age, were included. We excluded individuals with maternally inherited or unknown origin of 22q11.2 deletion $(n=7)$, given the uncertainty of the effect on in utero fetal development. ${ }^{15}$ Of the 123 subjects studied, most $(n=117$, 95.1\%) had confirmed or probable de novo deletions; 6 had confirmed or probable paternally inherited 22q11.2 deletions. The mean age of the 123 participants was $32.7(\mathrm{SD}=10.6)$ years; 56 (45.5\%) were male and 105 (85.4\%) were of European ancestry. There were four subjects (two with schizophrenia) with a positive family history of schizophrenia in a first degree relative.

\section{Clinical predictor variables}

Data on predictor variables were collected from comprehensive review of lifetime medical records, including birth and neonatal/pediatric records, and from pregnancy, birth, and developmental histories that were obtained in all but two cases from one or both parents, as previously described. ${ }^{19-21}$ We recorded birth weight in grams (median 2,977, range 1,361-4,710) and gestational age in weeks (median 40, range 30-43). We used a standard definition of prematurity ( $<37$ weeks of gestation) ${ }^{25}$ As a proxy measure of IUGR, we defined SGA conservatively as gestational age and sex-specific birth weight $<3$ rd percentile. ${ }^{26}$ In the latter subgroup we also included a well-documented clinical diagnosis of IUGR, made using standard criteria. ${ }^{27}$ For adjudication and comparison, we used population-based Canadian reference data for birth weight, gestational age, and percentiles. $^{28}$

Established risk factors for prematurity and SGA birth weight include maternal morbidities and substance use. ${ }^{29,30}$ In this study, we considered maternal history during pregnancy of hypertension (including preeclampsia), gestational diabetes, and any smoking, alcohol, and/or other substance use (Table 1). There were no cases with absence of prenatal care, history of assisted reproduction, or extremes of maternal age (i.e., $<17$ or $>40$ years $).{ }^{29,30}$

\section{Psychiatric diagnosis and outcome variables}

As before, ${ }^{4,13,20,21,23,24}$ we (A.S.B. or E.W.C.C.) performed expert psychiatric assessments of all participants using the Structured Clinical Interview for DSM-IV and information derived from direct patient interviews, collateral history from family members, and lifetime medical records. For the main outcome variables, there were 51 patients diagnosed with schizophrenia or schizoaffective disorder (collectively termed "schizophrenia"; mean age at onset, $20.2(\mathrm{SD}=5.6)$ years); the remaining 72 individuals with no psychotic illness formed the comparison group. Using our established protocol to assess intellect, including standard neurocognitive testing (intelligence quotient, IQ) for 118 patients, we determined that 61 participants had borderline to normal intellect and the remainder met criteria for intellectual disability (ID), at the mild $(n=53)$ or moderate/ severe $(n=9)$ level. $^{23}$

\section{Statistical analyses}

We first compared gestational ages and gestational age-corrected and sex-corrected birth weights in 22q11.2DS to a population-based Canadian reference, ${ }^{28}$ using $\chi^{2}$ tests, and calculated odds ratios (ORs) and 95\% confidence intervals (CIs). We compared the proportions born $<3 \mathrm{rd},<10$ th, and $<50$ th percentiles in the 22q11.2DS sample with those in the reference sample. ${ }^{28}$ We then constructed a logistic regression model to test whether adverse perinatal variables were associated with expression of schizophrenia in 22q11.2DS. Covariates considered were male sex, European ancestry, SGA birth weight, prematurity, and the presence of at least one 1 maternal factor (as above). We calculated adjusted ORs and 95\% CIs. Post hoc analyses included positive and negative predictive values (PPVs and NPVs, respectively) for significant predictor variables. For these calculations, we used the best estimate of the true prevalence of schizophrenia in 22q11.2DS (25\%). ${ }^{2,4,5}$ Other post hoc analyses

Table 1 Perinatal features associated with expression of schizophrenia in adults with 22q11.2 deletion syndrome

\begin{tabular}{|c|c|c|c|c|c|c|c|c|c|}
\hline \multirow{3}{*}{$\begin{array}{l}\text { Demographic and clinical predictors } \\
\text { Male sex }\end{array}$} & \multirow{3}{*}{$\begin{array}{c}\text { Total } \\
\boldsymbol{N} \\
56\end{array}$} & \multicolumn{2}{|c|}{$\begin{array}{c}\text { Schizophrenia } \\
\quad(n=51)\end{array}$} & \multicolumn{2}{|c|}{$\begin{array}{l}\text { No psychotic illness } \\
(n=72)^{\mathrm{b}}\end{array}$} & \multicolumn{4}{|c|}{$\begin{array}{c}\text { Logistic regression } \\
\text { analysis }^{\mathrm{a}}\end{array}$} \\
\hline & & $N$ & $\%$ & $N$ & $\%$ & OR & $\begin{array}{r}95 \\
\text { confid }\end{array}$ & $\begin{array}{l}\text { lald } \\
\text { limits }\end{array}$ & $P^{c}$ \\
\hline & & 26 & 51.0 & 30 & 41.7 & 1.29 & 0.59 & 2.81 & 0.52 \\
\hline European ancestry & 105 & 40 & 78.4 & 65 & 90.3 & 0.57 & 0.18 & 1.79 & 0.33 \\
\hline Maternal factors ${ }^{d}$ & 53 & 20 & 39.2 & 33 & 45.8 & 0.69 & 0.30 & 1.57 & 0.37 \\
\hline SGA $<3$ rd percentile & 26 & 16 & 31.4 & 10 & 13.9 & 3.52 & 1.34 & 9.22 & 0.01 \\
\hline Prematurity & 16 & 11 & 21.6 & 5 & 6.9 & 5.38 & 1.63 & 17.75 & 0.006 \\
\hline
\end{tabular}

OR, adjusted odds ratio; SGA, small for gestational age; significant findings shown in bold.

a Likelihood ratio test for the regression model: $\chi^{2}=16.72 ; \mathrm{df}=5, P=0.005$. ${ }^{\mathrm{b}}$ Mean age of nonpsychotic subjects with $22 \mathrm{q} 11.2 \mathrm{DS}$ was 29.8 (SD $=8.4$ ) years. Restricting the nonpsychotic group to the 47 subjects aged $\geq 25$ years at last follow-up and repeating the regression analysis yielded similar significant findings for the model $\left(\chi^{2}=14.91\right.$;

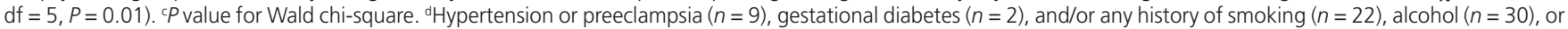
other substance use $(n=2)$ in pregnancy. 

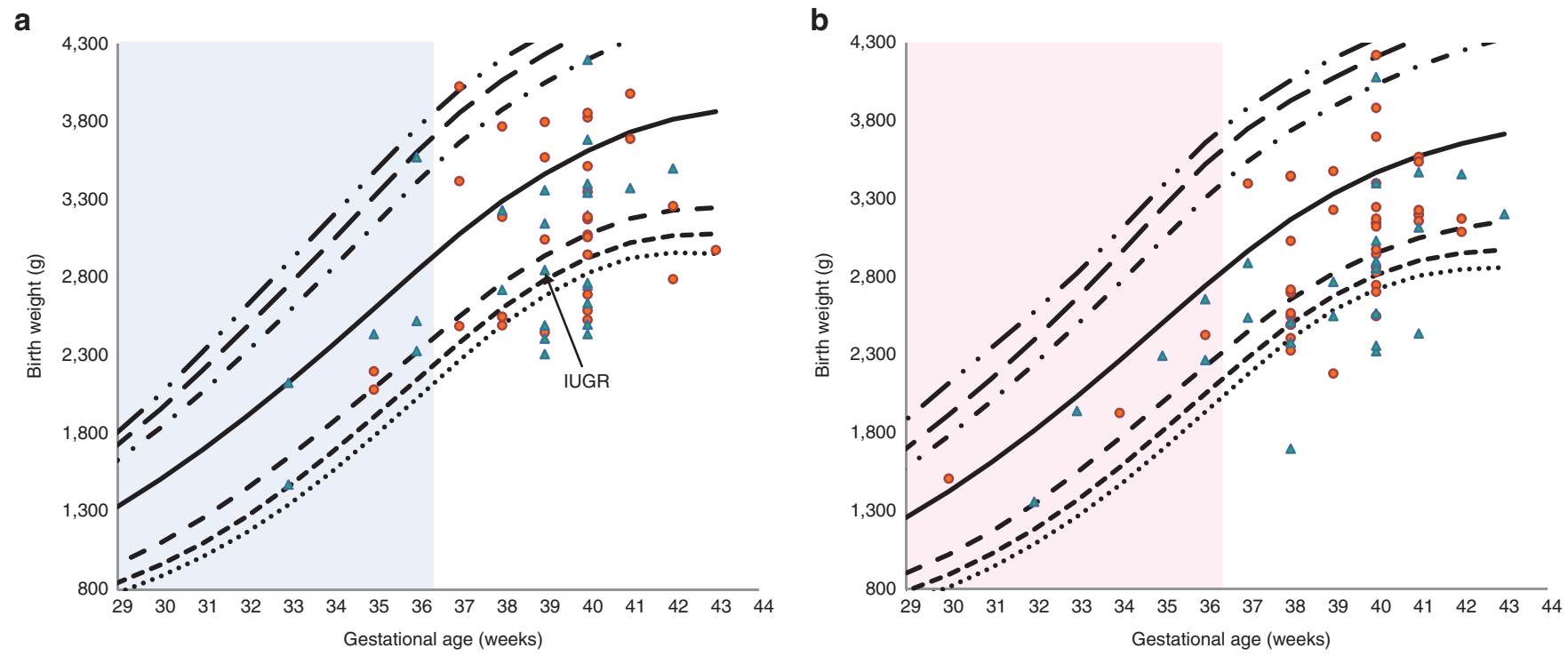

Schizophrenia $\bigcirc$ No psychotic illlness

Figure 1 Birth weight, gestational age, and schizophrenia status in 123 individuals with 22q11.2 deletion syndrome (22q11.2DS). Scatterplots of birth weight versus gestational age for $(\mathbf{a})$ male and (b) female singleton births with 22q11.2DS, using population normative percentile curves. ${ }^{28}$ From top to bottom, lines represent the 97th, 95th, 90th, 50th, 10th, 5th and 3rd percentiles, respectively. Shaded regions represent preterm births (<37 weeks gestation). One male case with a clinical diagnosis of intrauterine growth restriction (IUGR) is indicated by an arrow.

included examination of severity indicators (low IQ, clozapine use, younger age at onset) within the schizophrenia group. Between- and within-group calculations used $\chi^{2}$, Fisher's exact tests (FETs), or t-tests, as appropriate. Statistical analyses were performed using SAS 9.3 (SAS Institute, Cary, NC). Two-tailed $P$ values $<0.05$ were considered statistically significant.

\section{RESULTS}

\section{Prematurity and SGA birth weight are features of} 22q11.2DS

Individuals with 22q11.2DS in this cohort were significantly more likely to have been born premature or SGA than individuals in the general population (Figure 1). ${ }^{28}$ The prevalence of prematurity $(n=16,13.0 \%)$ in 22q11.2DS was greater than the population norm $\left(5.8 \% ; \chi^{2}=11.48, \mathrm{df}=1, P=0.0007 ; \mathrm{OR}=2.41\right.$, $95 \% \mathrm{CI}=1.33-4.10)$. Most were late preterm births-only five were $<34$ weeks (Figure 1 ). There were significantly more individuals with $22 \mathrm{q} 11.2 \mathrm{DS}$ born $<50$ th percentile $(n=102,82.9 \%$; $\left.\chi^{2}=53.33, \mathrm{df}=1, P<0.0001 ; \mathrm{OR}=4.86,95 \% \mathrm{CI}=3.01-8.18\right)$ and $<10$ th percentile $\left(n=48,39.0 \% ; \chi^{2}=115.05, \mathrm{df}=1\right.$, $P<0.0001 ; \mathrm{OR}=5.76,95 \% \mathrm{CI}=3.92-8.39)$ compared with the reference population. ${ }^{28}$ These findings were driven by the $25(20.3 \%)$ subjects born SGA $\left(\chi^{2}=126.72, \mathrm{df}=1, P<0.0001\right.$; $\mathrm{OR}=8.25,95 \% \mathrm{CI}=5.09-12.91)$, none of whom were preterm births (Figure 1). Restricting to the subgroup with no psychotic illness revealed a similar pattern (data not shown).

\section{Prematurity and SGA birth weight are predictors of schizophrenia within 22q11.2DS}

Consistent with our hypothesis, prematurity ( $\mathrm{OR}=5.38,95 \%$ $\mathrm{CI}=1.63-17.75)$ and SGA birth weight $(\mathrm{OR}=3.52,95 \%$
$\mathrm{CI}=1.34-9.22)$ were significant predictors of schizophrenia in a logistic regression model (Table 1). Maternal and other factors were not significant (Table 1), nor were there any significant interaction terms. Assuming a baseline prevalence of schizophrenia in 22q11.2DS of 25\%, ${ }^{2,45}$ the PPV for schizophrenia in the presence of either SGA birth weight or prematurity was $46 \%$ (sensitivity $53 \%$, specificity $79 \%$ ); the NPV in the absence of either feature was $83 \%$ (Figure 2).

Post hoc analyses showed there were no significant differences in maternal age at birth between those with and without schizophrenia $(26.7(\mathrm{SD}=4.5)$ years and $28.3(\mathrm{SD}=4.7)$ years, respectively; $t=1.83, \mathrm{df}=121, P=0.070)$. With respect to the four subjects with a positive family history of schizophrenia, the two in the schizophrenia group were born SGA, and the two in the nonpsychotic group were born neither SGA nor premature. Redoing the regression without these four subjects did not change the results (data not shown). Within each of the schizophrenia and nonpsychotic groups, comparing those with and without a history of prematurity or SGA birth weight, there were no significant differences in mean age or presence of serious congenital cardiac disease (data not shown).

\section{Prematurity and SGA birth weight may be associated with indicators of schizophrenia severity in 22q11.2DS}

Markers of severity in schizophrenia include greater cognitive impairment, treatment resistance (e.g., requiring clozapine ${ }^{24}$ ), and, to a lesser extent, younger age at onset. We conducted exploratory post hoc analyses of the potential impact of SGA birth weight and prematurity on schizophrenia disease severity. The mean IQ for those born SGA $(62.1, \mathrm{SD}=7.3)$ was significantly lower than for those not born SGA $(68.2, \mathrm{SD}=11.0$; 


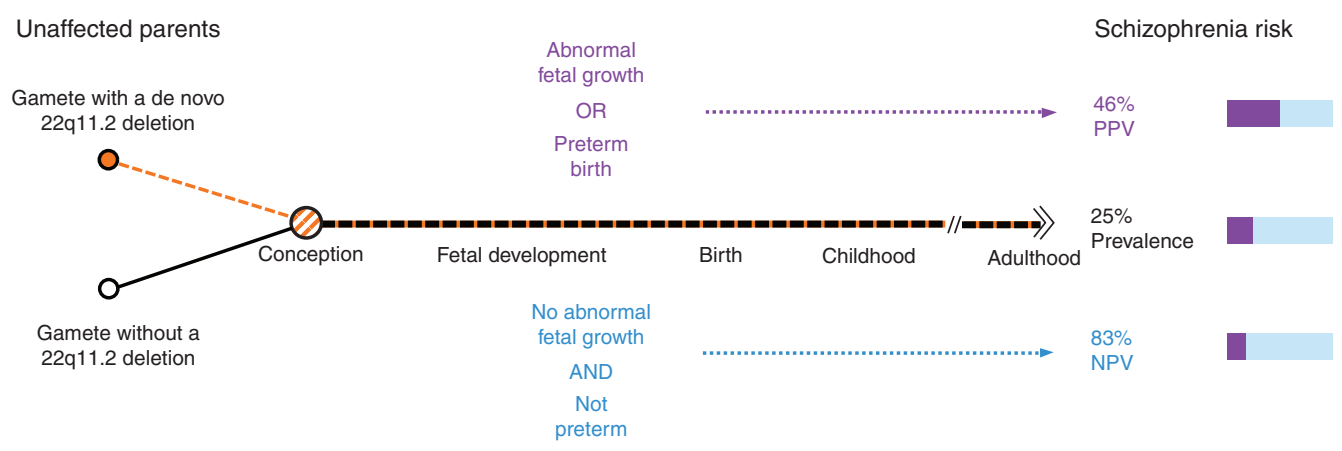

Figure 2 Predicting the expression of schizophrenia in individuals with 22q11.2 deletion syndrome (22q11.2DS). Diagram illustrating the proposed perinatal factors contributing to the risk for schizophrenia (purple bar) in 22q11.2DS, given the estimated 25\% baseline prevalence of schizophrenia conferred by the 22q11.2 deletion. The most common scenario of a de novo $22 q 11.2$ deletion is shown. NPV, negative predictive value; PPV, positive predictive value.

$t=2.03, \mathrm{df}=48, P=0.048)$. There was a similar trend for diagnosis of ID: $14(87.5 \%)$ of 16 born SGA versus $21(60.0 \%)$ of 35 not born SGA (FET $P=0.06$; OR $=4.67$ ). In contrast, there were no significant differences in mean IQ or ID diagnosis by SGA status within the nonpsychotic group (data not shown). There was a trend suggestive of an association between prematurity and treatment resistance: $6(54.5 \%)$ of 11 born preterm versus 9 (22.5\%) of 40 not born preterm had been treated with clozapine (FET $P=0.06$; OR = 4.13). Neither SGA birth weight nor prematurity had a significant impact on age at onset of schizophrenia (data not shown).

\section{DISCUSSION}

Consistent with our hypothesis, prematurity and SGA birth weight were associated with expression of schizophrenia within a molecularly defined population of individuals with 22q11.2DS, even after accounting for maternal factors. The results are consistent with previous findings in the general schizophrenia literature, ${ }^{7-10}$ but with noticeably greater effect sizes in this genetically more homogeneous population. These fetal growth and gestational factors augmented the baseline 25-fold increased risk for schizophrenia imparted by the 22q11.2 deletion. ${ }^{2,4}$ The accompanying high PPV and NPV indicate that abnormal fetal growth and gestation may be useful as markers for predicting expression of schizophrenia in 22q11.2DS (Figure 2). There are implications for pre- and postnatal testing and genetic counseling. These findings advance our understanding of the complex determinants of disease-specific expression in 22q11.2DS, with implications for other genomic disorders.

\section{SGA birth weight, IUGR, and aberrant neurodevelopmental trajectories}

Early neurodevelopmental factors are posited to be involved in expression of schizophrenia. ${ }^{6}$ The greater effect sizes seen in this study may be related to both the relative genetic homogeneity conferred by a 22q11.2 deletion and the stringent definition of SGA used ( $<3$ rd percentile). Severe SGA ( $<3$ rd percentile) may be more enriched for IUGR than the 3rd-10th-percentile group, where constitutionally small size (an apparent feature of 22q11.2DS) may comprise a greater proportion. ${ }^{26}$ Our findings suggest that the risk for schizophrenia related to fetal size may lie with IUGR, a pathological mechanism associated in the general population with elevated rates of infant mortality and neurodevelopmental impairment in infants and children. ${ }^{27,31,32}$ Precise timing of IUGR is often unknown. In the single subject in our sample clinically diagnosed with IUGR through serial ultrasounds, findings were normal at 15 weeks gestational age but showed progressively decelerating growth at 36 and 38.5 weeks.

\section{Molecular mechanisms and fetal factors}

While maternal factors are often considered paramount, factors that are innate to the fetus may contribute to IUGR as reflected by SGA birth weight. In this study, the fetuses, but not the mothers, had 22q11.2 deletions. We recently reported that offspring of mothers with 22q11.2DS were at increased risk for being born SGA only if they had inherited the deletion. ${ }^{15}$ Additional support for the likelihood that fetal genotype plays an important role in intrauterine growth comes from studies of other major (e.g., noninherited) genetic disorders. ${ }^{30}$ Epidemiologic studies have estimated that fetal genotype accounts for $\sim 37 \%$ of the variance in SGA risk $^{33}$ and for about $5 \%$ with respect to preterm birth. ${ }^{25}$ Thus genetic variation within and/or outside of the 22q11.2 deletion region that influences fetal development and the intrauterine environment may further increase the risk for schizophrenia that is imparted by gene dosage effects of the deletion itself. The pathologic mechanisms underlying these findings remain unknown but are deserving of future study. ${ }^{34,35}$

\section{Epidemiologic study design in the molecular era}

Results from population-based epidemiologic studies of genetically heterogeneous diseases like schizophrenia and autism spectrum disorders (ASD) conceal a wealth of genetic diversity. In large samples, small absolute differences can be statistically significant but have weak positive or negative predictive power. This study is proof-of-principle that restricting to a smaller but more homogeneous genetically defined subpopulation can result in robust, clinically relevant findings. Our results are consistent with the enticing possibility that certain established epidemiologic associations of modest effect size may be driven by specific individual genetic subtypes where the effect 
is much larger. There are broad implications for the design of epidemiologic studies in the molecular era. For example, in one recent population-based epidemiologic study of ASD, ${ }^{36}$ both SGA birth weight and prematurity were found to be risk factors of modest effect. Moreover, as in our study within the schizophrenia group, SGA birth weight was found to be associated with lower IQ within ASD. ${ }^{36}$ Our results suggest that molecular characterization of pathogenic copy-number variations using chromosomal microarray analysis (now the first-tier clinical diagnostic test in ASD) might allow for refinement of epidemiologic findings to the point of being clinically informative. ${ }^{37}$ Many more such predictions are promised by elucidation of all germline genomic variation using whole-genome sequencing.

\section{Strengths and limitations}

To our knowledge, this is the first study to rigorously investigate adverse perinatal outcomes as potential predictors of schizophrenia within 22q11.2DS. We employed a retrospective case-control design in the single largest and best characterized adult 22q11.2DS cohort in the world. We controlled for potential effects of demographic and maternal factors. That the overall rate of SGA birth weight reported in this study was comparable to those reported in independent 22q11.2DS cohorts provides confidence in the representativeness of our 22q11.2DS sample. ${ }^{16-18}$ However, this adult cohort is unique in its size and mean age, with definitive data available on psychotic disorder outcomes. We preferentially recruited individuals with schizophrenia. This enhanced the power to detect differences between the schizophrenia and nonpsychotic groups but did not bias the PPV and NPV calculations, which used established prevalence figures for schizophrenia in 22q11.2DS.

As for any retrospective study, available data restricted the sample size available. This may have limited our ability to detect significant effects for variables with small effect size, e.g., maternal factors ${ }^{30}$ as well as to assess other factors such as maternal stress which may be related to SGA birth weight, prematurity, and/or schizophrenia. Data from parental interviews raises the possibility of errors in recollection or biases in self-reporting, e.g., for maternal factors such as alcohol use. To help account for this, we supplemented interview data with birth records and a comprehensive review of neonatal and pediatric history. When comparing to the general population, we relied on published population norms for gestational age and gestational age- and sex-corrected birth weight. We did not investigate a comparison sample of individuals with schizophrenia and without 22q11.2 deletions. Birth weight is a commonly used but crude proxy for fetal growth that falls short of the ideal: serial ultrasound measurements throughout the course of the pregnancy.

In this study, nonpsychotic subjects with 22q11.2DS were mainly recruited through an adult congenital cardiac clinic. This meant that the majority of the nonpsychotic group had serious congenital cardiac defects and were on average eight years younger than the group with schizophrenia. We found no significant differences in age or prevalence of serious congenital cardiac defects by presence of SGA birth weight or prematurity within either the schizophrenia or nonpsychotic groups. Pediatric mortality in 22q11.2DS is rare. ${ }^{1,2}$ Nevertheless, we cannot rule out the possibility of a selective survival effect in which those born premature and/or SGA with 22q11.2DS and congenital cardiac defects preferentially died in the neonatal period or in childhood. Lastly, some individuals in the nonpsychotic group may go on to develop schizophrenia. This would be expected to have a limited effect on our findings, however, given that: (i) the average age of our nonpsychotic group was almost 10 years older than the mean age at onset of schizophrenia in 22q11.2DS, (ii) restricting the nonpsychotic group to subjects aged $\geq 25$ years and repeating the regression analysis yielded comparable significant findings for the model (Table 1), and (iii) a survival analysis to time to schizophrenia by SGA birth weight or prematurity, which accounted for the age of each participant, yielded comparable findings to the logistic regression models (data not shown).

\section{Conclusions and future directions}

In addition to standard indications for invasive prenatal testing, ${ }^{3,38}$ identification of $22 \mathrm{q} 11.2$ deletions is now possible by noninvasive prenatal testing that analyzes circulating cell free fetal DNA present in maternal serum ${ }^{39}$ as early as the first trimester. Future studies may now be able to prospectively measure fetal growth and outcome in unaffected mothers, where the fetus has been identified to have a 22q11.2 deletion. Ideally, follow-up of these offspring will continue long term for neurodevelopmental outcomes, including schizophrenia. Studies of the placenta and brain development in 22q11.2DS will further enhance our understanding of mechanisms. Identifying individuals at especially elevated risk for schizophrenia, as in 22q11.2DS, is increasingly a public health imperative. In the five years following a first psychotic episode, less than 15\% of patients with schizophrenia achieve full, sustained recovery. ${ }^{40}$ In understanding the mechanisms that mediate risk for schizophrenia in genetically predisposed individuals, there exists the possibility that modifiable factors may be identified and prevention strategies developed. ${ }^{6}$ These include potentially neuroprotective interventions applicable in utero and in the immediate postnatal period in the context of a fetus with $22 \mathrm{q} 11.2$ deletion.

In this study, we found that prematurity and birth weight $<3$ rd percentile for sex and gestational age are significant risk factors for the development of schizophrenia in individuals with 22q11.2DS. These results indicate that abnormal fetal growth and gestation can act as clinically relevant factors in expression of schizophrenia. While there is a need for replication, it is to be hoped that such findings may assist both clinicians and families in developing a more refined risk assessment of schizophrenia in 22q11.2DS. These findings reflect the power of the 22q11.2DS model for identifying potential predictive factors for expression of schizophrenia, in addition to the 22q11.2 deletion itself. Our results also represent progress more generally towards addressing one of the great questions in human genetics and neuroscience-the reduced penetrance and variable expression of copy-number variations with respect to 
neuropsychiatric disease. New perspectives on the role of fetal genetics in obstetrical practice could have important impact on individualized management, in an era of changing prenatal molecular screening and diagnosis.

\section{ACKNOWLEDGMENTS}

The authors thank the patients and their families for their participation; colleagues for referring patients; and research assistants, students, and fellows who assisted in the collection of data for the study. This work was supported by the Canadian Institutes of Health Research (CIHR) (MOP-313331 and MOP-111238 to A.S.B.), the Mach-Gaensslen Foundation of Canada (L.V.), a Brain Canada Mental Health Training Award (N.J.B.), and MD/PhD Studentships from the CIHR and the McLaughlin Centre (G.C.). A.S.B. holds the Canada Research Chair in Schizophrenia Genetics and Genomic Disorders, and the Dalglish Chair in 22q11.2 Deletion Syndrome.

\section{DISCLOSURE}

The authors declare no conflict of interest.

\section{REFERENCES}

1. Bassett AS, McDonald-McGinn DM, Devriendt K, et al.; International 22q11.2 Deletion Syndrome Consortium. Practical guidelines for managing patients with 22q11.2 deletion syndrome. J Pediatr 2011;159:332-9.e1.

2. Fung WL, Butcher NJ, Costain G, et al. Practical guidelines for managing adults with 22q11.2 deletion syndrome. Genet Med 2015; e-pub ahead of print 8 January 2015.

3. Costain G, McDonald-McGinn DM, Bassett AS. Prenatal genetic testing with chromosomal microarray analysis identifies major risk variants for schizophrenia and other later-onset disorders. Am J Psychiatry 2013;170:1498.

4. Fung WL, McEvilly R, Fong J, Silversides C, Chow E, Bassett A. Elevated prevalence of generalized anxiety disorder in adults with $22 q 11.2$ deletion syndrome. Am J Psychiatry 2010;167:998.

5. Schneider M, Debbané M, Bassett AS, et al.; International Consortium on Brain and Behavior in 22q11.2 Deletion Syndrome. Psychiatric disorders from childhood to adulthood in 22q11.2 deletion syndrome: results from the International Consortium on Brain and Behavior in 22q11.2 Deletion Syndrome. Am J Psychiatry 2014;171:627-639.

6. Insel TR. Rethinking schizophrenia. Nature 2010;468:187-193.

7. Kunugi H, Nanko S, Murray RM. Obstetric complications and schizophrenia: prenatal underdevelopment and subsequent neurodevelopmental impairment. Br J Psychiatry Supp/ 2001;40:s25-s29.

8. Knud Larsen J, Bendsen BB, Foldager L, Munk-Jørgensen P. Prematurity and low birth weight as risk factors for the development of affective disorder, especially depression and schizophrenia: a register study. Acta Neuropsychiatr 2010;22:284-291.

9. Nosarti C, Reichenberg A, Murray RM, et al. Preterm birth and psychiatric disorders in young adult life. Arch Gen Psychiatry 2012;69:E1-E8.

10. Nielsen PR, Mortensen PB, Dalman C, et al. Fetal growth and schizophrenia: a nested case-control and case-sibling study. Schizophr Bull 2013;39:1337-1342.

11. Kugelman A, Colin AA. Late preterm infants: near term but still in a critical developmental time period. Pediatrics 2013;132:741-751.

12. Levine TA, Grunau RE, McAuliffe FM, Pinnamaneni R, Foran A, Alderdice FA. Early childhood neurodevelopment after intrauterine growth restriction: a systematic review. Pediatrics 2015;135:126-141.

13. Chow EW, Husted J, Weksberg R, Bassett AS. Postmaturity in a genetic subtype of schizophrenia. Acta Psychiatr Scand 2003;108:260-268.

14. Forsyth JK, Ellman LM, Tanskanen A, et al. Genetic risk for schizophrenia, obstetric complications, and adolescent school outcome: evidence for gene-environment interaction. Schizophr Bull 2013;39:1067-1076.

15. Chan C, Costain G, Ogura L, et al. Reproductive health issues for adults with a common genomic disorder: 22q11.2 deletion syndrome. J Genet Couns 2015; e-pub ahead of print 13 January 2015.
16. Reynaud R, Derain-Court J, Braunstein D, et al. Auxological evaluation in patients with a 22q11.2 microdeletion syndrome: normal prevalence of obesity and neonatal length and gender influence on body mass index evolution. Horm Res Paediatr 2011;76:172-177.

17. Besseau-Ayasse J, Violle-Poirsier C, Bazin A, et al. A French collaborative survey of 272 fetuses with 22q11.2 deletion: ultrasound findings, fetal autopsies and pregnancy outcomes. Prenat Diagn 2014;34:424-430.

18. Tarquinio DC, Jones MC, Jones KL, Bird LM. Growth charts for $22 q 11$ deletion syndrome. Am J Med Genet A 2012;158A:2672-2681.

19. Bassett AS, Chow EW, Husted J, et al. Clinical features of 78 adults with $22 q 11$ Deletion Syndrome. Am J Med Genet A 2005;138:307-313.

20. Costain G, Chow EW, Silversides CK, Bassett AS. Sex differences in reproductive fitness contribute to preferential maternal transmission of $22 q 11.2$ deletions. J Med Genet 2011:48:819-824.

21. Cheung EN, George SR, Andrade DM, Chow EW, Silversides CK, Bassett AS. Neonatal hypocalcemia, neonatal seizures, and intellectual disability in 22q11.2 deletion syndrome. Genet Med 2014;16:40-44.

22. Bassett AS, Marshall CR, Lionel AC, Chow EW, Scherer SW. Copy number variations and risk for schizophrenia in 22q11.2 deletion syndrome. Hum Mol Genet 2008; 17:4045-4053.

23. Butcher NJ, Chow EW, Costain G, Karas D, Ho A, Bassett AS. Functional outcomes of adults with 22q11.2 deletion syndrome. Genet Med 2012;14: $836-843$.

24. Butcher NJ, Fung WL, Fitzpatrick $L$, et al. Response to clozapine in a clinically identifiable subtype of schizophrenia. Br J Psychiatry 2015; e-pub ahead of print 5 March 2015

25. Svensson AC, Sandin S, Cnattingius S, et al. Maternal effects for preterm birth: a genetic epidemiologic study of 630,000 families. Am J Epidemiol 2009;170:1365-1372.

26. Campbell MK, Cartier S, Xie B, Kouniakis G, Huang W, Han V. Determinants of small for gestational age birth at term. Paediatr Perinat Epidemiol 2012;26: 525-533.

27. Resnik R. Intrauterine growth restriction. Obstet Gyneco/ 2002;99:490-496.

28. Kramer MS, Platt RW, Wen SW, et al.; Fetal/Infant Health Study Group of the Canadian Perinatal Surveillance System. A new and improved populationbased Canadian reference for birth weight for gestational age. Pediatrics 2001;108:E35

29. Goldenberg RL, Culhane JF, lams JD, Romero R. Epidemiology and causes of preterm birth. Lancet 2008;371:75-84.

30. Lee PA, Chernausek SD, Hokken-Koelega AC, Czernichow P; International Small for Gestational Age Advisory Board. International Small for Gestational Age Advisory Board consensus development conference statement: management of short children born small for gestational age, April 24-October 1, 2001. Pediatrics 2003;111(6 Pt 1):1253-1261.

31. Lausman A, Kingdom J, Gagnon R, et al.; Maternal Fetal Medicine Committee. Intrauterine growth restriction: screening, diagnosis, and management. J Obstet Gynaecol Can 2013;35:741-757.

32. von Beckerath AK, Kollmann M, Rotky-Fast C, Karpf E, Lang U, Klaritsch P. Perinatal complications and long-term neurodevelopmental outcome of infants with intrauterine growth restriction. Am J Obstet Gynecol 2013;208: 130.e1-130.e6.

33. Svensson AC, Pawitan $Y$, Cnattingius S, Reilly M, Lichtenstein P. Familial aggregation of small-for-gestational-age births: the importance of fetal genetic effects. Am J Obstet Gynecol 2006; 194:475-479.

34. Brzustowicz LM, Bassett AS. miRNA-mediated risk for schizophrenia in 22q11.2 deletion syndrome. Front Genet 2012;3:291

35. Merico D, Costain G, Butcher NJ, et al. MicroRNA Dysregulation, Gene Networks, and Risk for Schizophrenia in 22q11.2 Deletion Syndrome. Front Neurol 2014;5:238.

36. Abel KM, Dalman C, Svensson AC, et al. Deviance in fetal growth and risk of autism spectrum disorder. Am J Psychiatry 2013;170:391-398.

37. Miller DT, Adam MP, Aradhya S, et al. Consensus statement: chromosomal microarray is a first-tier clinical diagnostic test for individuals with developmental disabilities or congenital anomalies. Am J Hum Genet 2010;86:749-764.

38. Wapner RJ, Martin CL, Levy B, et al. Chromosomal microarray versus karyotyping for prenatal diagnosis. N Engl J Med 2012;367:2175-2184.

39. Jensen TJ, Dzakula Z, Deciu C, van den Boom D, Ehrich M. Detection of microdeletion $22 q 11.2$ in a fetus by next-generation sequencing of maternal plasma. Clin Chem 2012:58:1148-1151.

40. Robinson DG, Woerner MG, McMeniman M, Mendelowitz A, Bilder RM. Symptomatic and functional recovery from a first episode of schizophrenia or schizoaffective disorder. Am J Psychiatry 2004;161:473-479. 